\title{
Accurate and Efficient Evaluation of Bit Error Rate for Dynamic Directional Modulation for Standard Modulation Schemes
}

\author{
Josep Parrón ${ }^{1, * \mathbb{D}}$, Edith Cabrera-Hernandez ${ }^{1}$ (D) and Alan Tennant ${ }^{2}$ \\ 1 Department of Telecommunications and Systems Engineering, Universitat Autonoma de Barcelona, \\ 08193 Bellaterra, Spain; edithannette.cabrera@uab.cat \\ 2 Department of Electronic and Electrical Engineering, The University of Sheffield, Sheffield S1 4ET, UK; \\ a.tennant@sheffield.ac.uk \\ * Correspondence: josep.parron@uab.cat
}

Citation: Parrón, J.;

Cabrera-Hernandez, E.; Tennant, A. Accurate and Efficient Evaluation of Bit Error Rate for Dynamic

Directional Modulation for Standard Modulation Schemes. Electronics 2021, 10, 776. https://doi.org/10.3390/ electronics10070776

Academic Editor: Konstantinos Peppas

Received: 27 January 2021

Accepted: 23 March 2021

Published: 25 March 2021

Publisher's Note: MDPI stays neutral with regard to jurisdictional claims in published maps and institutional affiliations.

Copyright: (c) 2021 by the authors. Licensee MDPI, Basel, Switzerland. This article is an open access article distributed under the terms and conditions of the Creative Commons Attribution (CC BY) license (https:// creativecommons.org/licenses/by/ $4.0 /)$.

\begin{abstract}
Directional modulation (DM) has been proposed as a technique to enhance physical layer security of wireless transmissions. In DM, the improvement of security is achieved by increasing the transmitted power in such a way that the bit error rate (BER) is degraded in the observation angles out of the desired secure direction. The performance of DM in terms of BER is typically evaluated by transmitting a stream of symbols for every observation angle, but this approach can be time consuming. In this communication, we propose an approach to evaluate, accurately and efficiently, the BER of dynamic DM (DDM) for standard modulation schemes. Several DDM configurations will be tested to illustrate the benefits and limitations of the evaluation method. The proposed approach is also used to present a non-iterative DDM synthesis with restrictions in the BER response.
\end{abstract}

Keywords: directional modulation; phased arrays; bit error rate; secure communication

\section{Introduction}

In wireless transmissions, the absence of a physical boundary that limits the wave propagation makes transmitted information highly vulnerable to interception by potential eavesdroppers [1]. Traditionally, cryptographic techniques have been applied at the higher protocol layers to overcome this limitation; however, the exchange of the private key in keybased methods also requires a secure channel that may not be available [2]. Additionally, the relentless growth of computational power is making it possible to break ciphers that were considered virtually unbreakable in the past [3]. In this sense, an additional level of security will be achieved if encryption is also performed in the physical layer where the raw interchange of information takes place.

Physical layer security (PLS) exploits intrinsic characteristics of the wireless channel to limit the amount of information that can be extracted at bit level by a potential eavesdropper [4]. A number of PLS schemes have been developed and published in the open literature [4-6]. Directional modulation (DM) is a keyless PLS transmitting technique that can be classified as an artificial noise (AN) approach [7]. DM is typically based on antenna arrays that generate multiple transmission radiation patterns. These radiation patterns are chosen to produce the desired constellation in the selected secure observation angle and, at the same time, a distorted or noisy version of the constellation in the other observation angles [8]. DM is termed static (SDM) when the distortion does not change with time and dynamic (DDM) when the distortion changes at the symbol rate [9].

The generation of DM inherently entails an increase in the transmitted power with respect to a conventional modulation scheme that transmits the same desired constellation in every observation angle [9]. The ratio between the power transmitted in DM and a conventional modulation scheme defines a power efficiency $\left(P E_{D M}\right)$ that allows for comparison between different DM implementations. For a conventional modulation, 
$P E_{D M}=100 \%$ and a decrease in this efficiency is typically related to an improvement in the transmission security [9].

Bit error rate (BER), as a function of the observation angle, is another figure of merit that is typically used to evaluate the degree of security provided by DM [9]. Hence, a DM scheme is usually designed with two main goals: (1) to decrease BER in the side lobes of the conventional modulation radiation pattern and (2) to narrow the main lobe beam width where the signal is demodulated with a BER below a given threshold (information beam width). It is important to acknowledge that BER and $P E_{D M}$ are not uniquely related, so two DM implementations with the same $P E_{D M}$ can show a substantially different response in terms of BER.

\subsection{Related Work}

In the literature, we can find different implementations of DM [10-32]. These solutions differ in the architecture of the array feeding network and the synthesis of the weights that feed the antennas.

Regarding the feeding networks, we can distinguish between those that use switches [10,13], phase shifters $[11,12,20,31]$, phase shifters with switches [17,21,29], vector modulators (reconfigurable amplitude and phase) [18,19,22-26,30,32], IQ data separately encoded transmitters [14,27], retrodirective array [28] and a Fourier Rotman lens [15,16].

Some of the reported architectures $[14-16,27,28]$ do not require a synthesis method for the antenna weights since the feeding network generates DM straightforwardly. Among the reported synthesis methods, we can find some that make use of brute force and explore all the possible combinations allowed by the architecture by searching for those that generate the desired constellation in the secure direction $[10,13,20]$. Other authors utilize optimization algorithms to obtain the antenna weights; some examples are genetic [11,12], simulated annealing [17], particle swarm [18], differential evolution [29] and convex optimization [30-32]. In these cases, the optimization procedure tries to minimize a cost function subject to some constraints that are related to the architecture limitations, $P E_{D M}$ and/or BER in certain observation angles. We can also find synthesis methods inspired by concepts [24] or methods that try to minimize the variations of BER response with respect to errors in the estimation of the secure observation angle [25]. Finally, there is the orthogonal vector approach proposed in [19] as a general framework for the synthesis of $\mathrm{DM}$. The orthogonal vector approach was later adapted to include restrictions in the BER response [18], in the radiation patterns [22] and in the orthogonal artificial noise injected in some observation angles [23].

All the above-mentioned methods need to evaluate BER during the design process and / or at the end of the synthesis in order to determine the level of security that can be expected from the DM transmission. BER evaluation techniques used in the literature are summarized in Table 1. In some cases [11-13,18], the evaluation of BER is carried out, for every observation angle, using approximations that only apply to quadrature phase shift keying (QPSK) modulation and zero-mean artificial orthogonal Gaussian noise. However, most of the evaluation techniques [10-19,21-32] use the time-consuming transmission of a stream of $10^{6}-10^{7}$ symbols in a channel with additive white Gaussian noise (AWGN), since it can be applied to SDM and DDM with zero-mean artificial orthogonal noise.

Table 1. Directional modulation (DM) system performance assessment: bit error rate (BER) evaluation techniques

\begin{tabular}{ccc}
\hline & Closed-Form Equation [11-13,18] & Data Stream Simulation [10-19,21-32] \\
\hline Static DM & Only QPSK & Yes \\
\hline Dynamic DM: zero-mean Gaussian orthogonal artificial noise & Only QPSK & Yes \\
\hline Dynamic DM: zero-mean non-Gaussian orthogonal artificial noise & No & Yes \\
\hline Computation time & Low & High \\
\hline
\end{tabular}




\subsection{Our Contributions}

This paper proposes a novel and new approach to evaluate, accurately and efficiently, BER of DDM for standard modulations with zero-mean orthogonal artificial noise. It will be shown that, under certain conditions that are easily met in real scenarios, BER can be derived without the need for transmitting a stream of symbols. This approach also leads to an improvement to the existing DDM synthesis techniques by equating the BER response to the amount of artificial noise injected at particular observation angles. This approach removes the need to carry out an iterative or optimization process.

This document is structured as follows: Section 2 reviews the orthogonal vector approach for generating DDM; Section 3 derives an expression for the average signal-to-noise ratio in a DDM transmission as a function of the observation angle. In Section 4, the average signalto-noise ratio is used to evaluate BER efficiently for different modulation schemes and DDM configurations. Limitations of the approach are also discussed in Section 4. In Section 5, we propose a novel non-iterative BER-driven DDM synthesis by applying the concepts of previous sections. Finally, conclusions are summarized in Section 6.

\section{Orthogonal Vector Approach for Dynamic Directional Modulation}

Let us consider a conventional array in free space with $N$ omnidirectional antennas distributed along the $\mathrm{x}$-axis (Figure 1). A standard constellation of $M$ symbols will be transmitted towards the observation angle $\phi_{0}$ of the XY plane $\left(\theta=90^{\circ}\right)$. In a conventional modulation system, the radiation pattern used in the transmission of symbol $d_{m}$ is given by

$$
s_{m \_c o n v}(\phi)=\sum_{n=1}^{N} B_{m n \_c o n v} A E P_{n}(\phi)=\mathbf{A E P}(\phi)^{T} \cdot \mathbf{B}_{m \_c o n v} \quad m \in\{1 \ldots M\} .
$$

where column vector $\mathbf{A E P}(\phi)$ contains the active element pattern [33] of each antenna in the array for angle $\phi,{ }^{T}$ is the transpose operator and $\mathbf{B}_{m \_}$conv is a column vector with the antenna weights defined as

$$
\mathbf{B}_{m \_c o n v}=d_{m} \mathbf{A E P}\left(\phi_{0}\right)^{*} \quad m \in\{1 \ldots M\}
$$

with * denoting the conjugate operator.

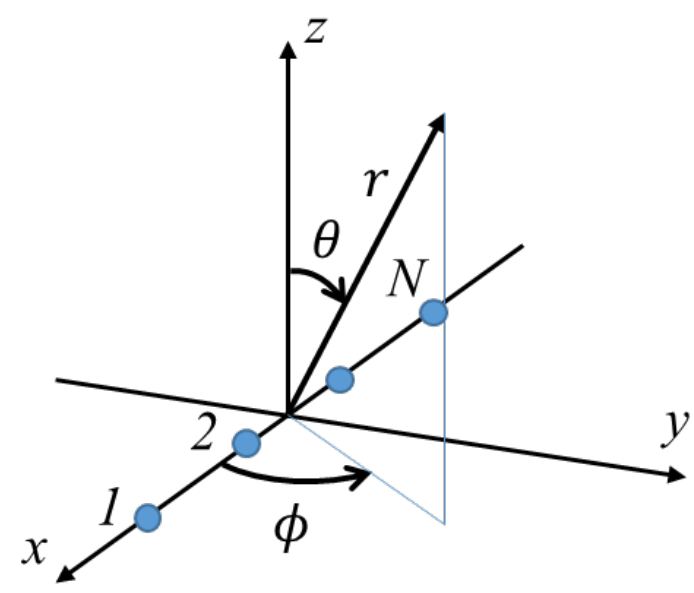

Figure 1. Array of $N$ omnidirectional antennas along the x-axis.

For convenience, and without loss of generality, symbols $d_{m}$ are normalized such as

$$
\frac{1}{M} \sum_{m=1}^{M}\left\|\mathbf{B}_{m \_c o n v}\right\|^{2}=1
$$

where $\|\cdot\|$ is the norm of a vector. 
In DDM, each symbol $d_{m}$ is transmitted using $P$ different radiation patterns. For that purpose, antenna weights $\mathbf{B}_{m p_{-} D D M}$ are obtained adding a vector of artificial noise $\mathbf{W}_{m p}$ to $\mathbf{B}_{m \_ \text {conv }}[19]$

$$
\begin{gathered}
s_{m p_{-} D D M}(\phi)=\mathbf{A E P}(\phi)^{T} \cdot \mathbf{B}_{m p \_D D M}=\mathbf{A E P}(\phi)^{T} \cdot\left(\mathbf{B}_{m_{-} c o n v}+\mathbf{W}_{m p}\right) \\
m \in\{1 \ldots M\} \quad p \in\{1 \ldots P\} .
\end{gathered}
$$

According to the orthogonal vector approach [19], vectors $\mathbf{W}_{m p}$ are chosen randomly to satisfy

$$
s_{m p \_D D M}\left(\phi_{0}\right)=s_{m_{-} \text {conv }}\left(\phi_{0}\right) \quad m \in\{1 \ldots M\}, \quad p \in\{1 \ldots P\} ;
$$

therefore, vectors $\mathbf{W}_{m p}$ must belong to the null space of $\operatorname{AEP}\left(\phi_{0}\right)$ (artificial orthogonal noise)

$$
\operatorname{AEP}\left(\phi_{0}\right)^{T} \cdot \mathbf{W}_{m p}=0 \quad m \in\{1 \ldots M\}, \quad p \in\{1 \ldots P\} .
$$

As a consequence, a noisy constellation is transmitted along the observation angles different to $\phi_{0}$. It must be noted that SDM is obtained for DDM with $P=1$; in this case, a distorted version of the constellation is obtained.

From the comparison of (1) and (4), it can be concluded that the implementation of DDM requires the addition of extra power to the transmission, so the power efficiency of $\operatorname{DDM}\left(P E_{D D M}\right)$ is defined as [9]

$$
P E_{D D M}=\frac{\frac{1}{M} \sum_{m=1}^{M}\left\|\mathbf{B}_{m \_c o n v}\right\|^{2}}{\frac{1}{M P} \sum_{p=1}^{P} \sum_{m=1}^{M}\left\|\mathbf{B}_{m \_c o n v}+\mathbf{W}_{m p}\right\|^{2}},
$$

and taking into consideration (3) and (6), it becomes

$$
P E_{D D M}=\frac{1}{1+\frac{1}{M P} \sum_{p=1}^{P} \sum_{m=1}^{M}\left\|\mathbf{W}_{m p}\right\|^{2}} .
$$

\section{Derivation of Average SNR for Dynamic Directional Modulation}

Next, we establish a connection between the signal-to-noise ratio (SNR) that can be observed in a given transmission angle and the artificial orthogonal noise injected into that direction. The average radiation pattern transmitted with $\operatorname{DDM}\left(r p_{a v_{-} D D M}(\phi)\right)$ is obtained as

$$
r p_{a v_{-} D D M}(\phi)=\frac{1}{M P} \sum_{p=1}^{P} \sum_{m=1}^{M}\left|s_{m p \_D D M}(\phi)\right|^{2} .
$$

Similarly, the average radiation pattern transmitted in the conventional modulation $\left(r p_{a v \_c o n v}(\phi)\right)$ is given by

$$
r p_{a v \_c o n v}(\phi)=\frac{1}{M} \sum_{m=1}^{M}\left|s_{m \_c o n v}(\phi)\right|^{2} .
$$

Then, we can compute the average radiation pattern of the injected artificial orthogonal noise $\left(r p_{a v_{-} W}(\phi)\right)$ as

$$
r p_{a v_{-} W}(\phi)=r p_{a v \_D D M}(\phi)-r p_{a v_{-} c o n v}(\phi) .
$$

Now, we define an average signal-to-noise ratio that depends on the observation angle as

$$
S N R_{a v}(\phi)=\frac{r p_{a v \_c o n v}(\phi)}{r p_{a v_{-} W}(\phi)+N_{A W G N}}
$$

where we have also included the contribution of additive white Gaussian noise $N_{A W G N}$ in the communication, assuming that it is uncorrelated to the artificial noise and uniform 
for all observation angles. At the secure observation angle, $S N R_{a v}\left(\phi_{0}\right)$ coincides with $\operatorname{SNR}\left(\phi_{0}\right)$ that would be obtained in a conventional modulation.

\section{Evaluation of BER for Dynamic Directional Modulation}

In this section, we use (12) to evaluate BER efficiently and accurately when zero mean artificial noise is injected to generate DDM. It must be pointed out that, in (12), no assumption has been made regarding the transmitted constellation; therefore, this approach applies for any standard modulation scheme.

\subsection{Zero Mean Artificial Gaussian Noise: Results and Discussion}

A straightforward choice for the zero mean artificial noise is to follow a Gaussian distribution. In that case, we can compute $S N R_{a v}(\phi)$ through (12) and evaluate BER using well-known expressions that can be found in the literature $[34,35]$ or the berawgn MATLAB function [36].

Configurations with different modulation schemes, $\operatorname{SNR}\left(\phi_{0}\right)$ and $P E_{D D M}$ were tested, and the average radiation patterns of (9)-(11) are plotted in Figure 2. The artificial noise average patterns $\left(r p_{a v_{-} W}(\phi)\right)$ of Figure 2 reveal that the synthesis strategy proposed in [19] spreads the artificial noise uniformly in all the observation angles outside of the main beam. As a consequence, $S N R_{a v}(\phi)$ in the side lobes and the information beam width cannot be adjusted independently. This strategy is also inefficient in terms of $P E_{D D M}$, since artificial noise is transmitted along some directions, such as the radiation nulls of the conventional radiation pattern, where no information is sent.

Figure 3 shows the BER computed with our approach (for $p \gg 1$ ) and expressions in Table 2 compared to the BER evaluated through the transmission of $10^{6}$ symbols following the procedures described in $[9,19]$; all the results show very good agreement. Considering that in $[9,19]$, eavesdroppers follow a training stage before demodulating, the scenario under evaluation is very unfavorable from the point of view of security. Although the information beam width also depends on the modulation scheme, in Figure 3, we can also check that the narrower information beam width corresponds to the scenario with the lowest $P E_{D D M}$ in spite of having the highest $S N R\left(\phi_{0}\right)$ (Figure 3c).

Figure 4 compares BER evaluated using a stream of $10^{6}$ symbols to our approach as the number of available radiation patterns per symbol $P$ increases. Obviously, for SDM $(P=1)$, it cannot be assumed that the added artificial noise follows a Gaussian distribution, therefore, we can find differences between both methods in the side lobe region and in the edges of the information beam. However, both BER evaluations methods converge rapidly as $P$ increases (DDM), and four different radiation patterns per transmitted symbol are enough to produce similar results.

Table 2. BER for uncoded data over AWGN channels [34,35].

\begin{tabular}{cccc}
\hline Modulation Scheme & QPSK & 16-QAM & 8-PSK \\
\hline$B E R(\phi)$ & $Q\left(\sqrt{S N R_{a v}(\phi)}\right)$ & $\frac{3}{4} Q\left(\sqrt{\frac{1}{5} S N R_{a v}(\phi)}\right)$ & $\frac{2}{3} Q\left(\sin \left(\frac{\pi}{8}\right) \sqrt{2 S N R_{a v}(\phi)}\right)$ \\
\hline
\end{tabular}




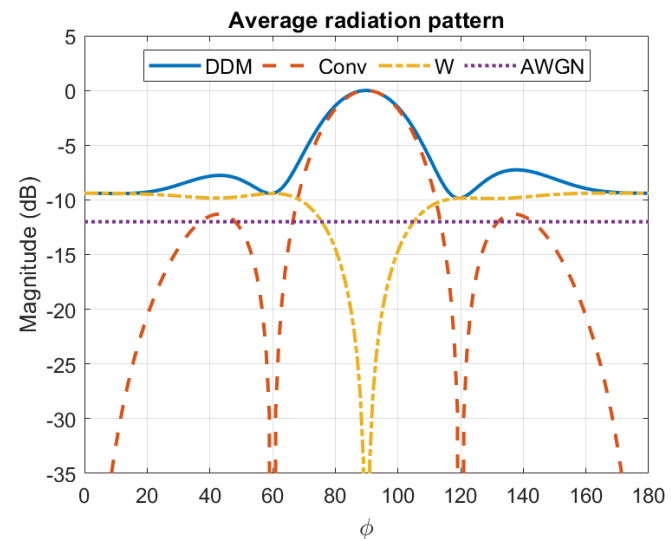

(a)

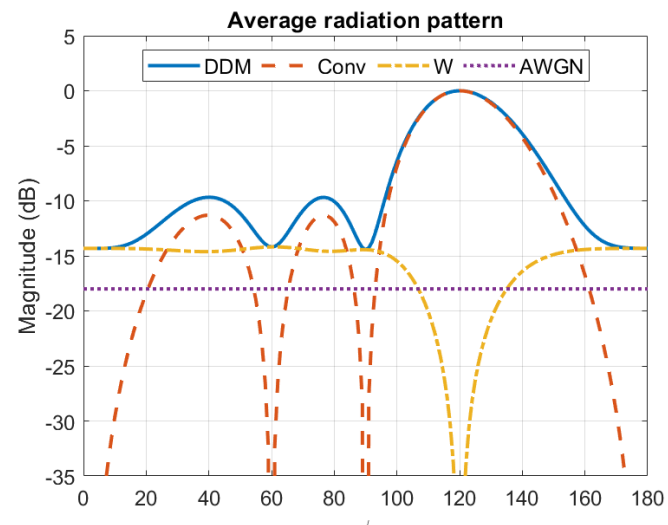

(b)

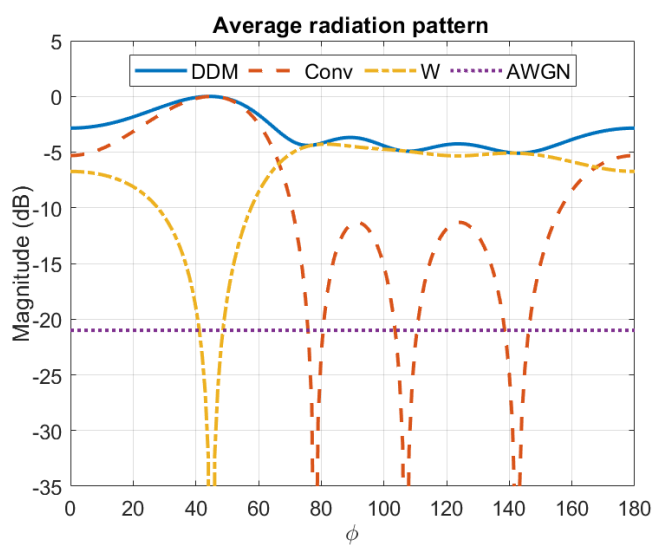

(c)

Figure 2. Array of four omnidirectional antennas along $x$-axis, spacing $\lambda / 2$. Average radiation patterns in $X Y$ plane for dynamic directional modulation (DDM) $(P=64)$. Zero mean artificial Gaussian noise. Only half-plane is plotted due to symmetry. (a) QPSK modulation, $\phi_{0}=90^{\circ}, \operatorname{SNR}\left(\phi_{0}\right)=12 \mathrm{~dB}$, $P E_{D D M}=75 \%$. (b) 16-QAM modulation, $\phi_{0}=120^{\circ}, \operatorname{SNR}\left(\phi_{0}\right)=18 \mathrm{~dB}, P E_{D D M}=90 \%$. (c) 8 -PSK modulation, $\phi_{0}=45^{\circ}, \operatorname{SNR}\left(\phi_{0}\right)=21 \mathrm{~dB}, P E_{D D M}=50 \%$. 


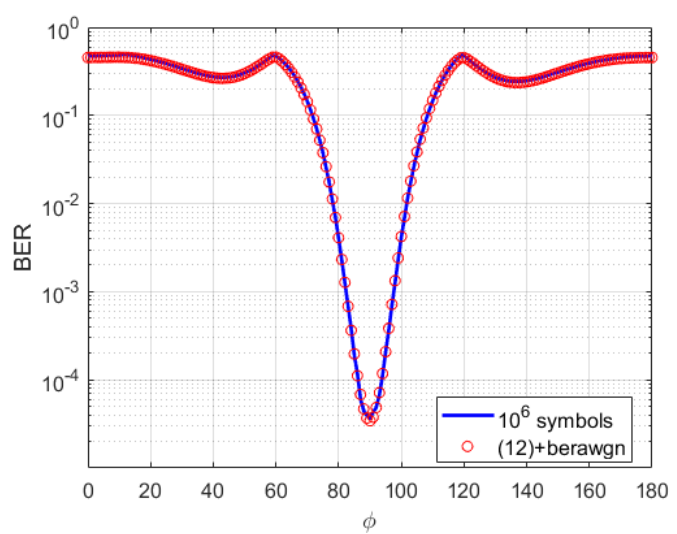

(a)

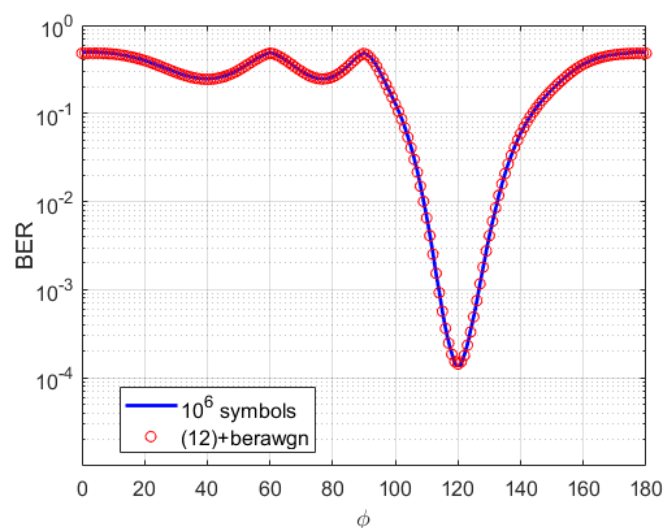

(b)

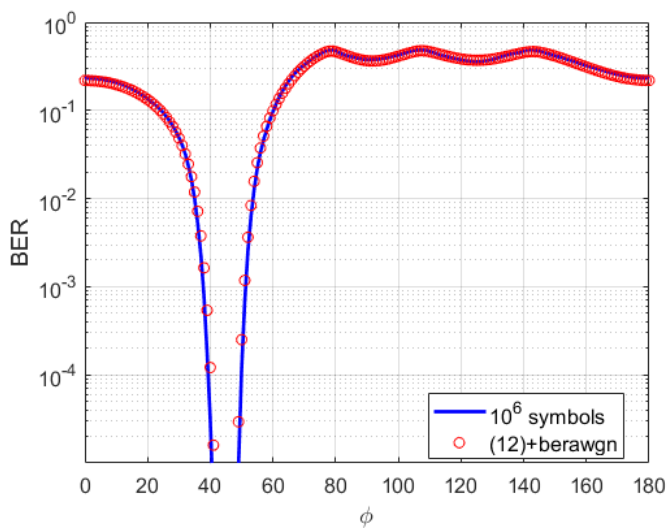

(c)

Figure 3. Array of four omnidirectional antennas along $x$-axis, spacing $\lambda / 2$. BER in $X Y$ plane for $\operatorname{DDM}(P=64)$. Zero mean artificial Gaussian noise. Only half-plane is plotted due to symmetry. (a) QPSK modulation, $\phi_{0}=90^{\circ}$, signal-to-noise ratio $(\mathrm{SNR})\left(\phi_{0}\right)=12 \mathrm{~dB}, P E_{D D M}=75 \%$. (b) 16 QAM modulation, $\phi_{0}=120^{\circ}, \operatorname{SNR}\left(\phi_{0}\right)=18 \mathrm{~dB}, P E_{D D M}=90 \%$. (c) 8-PSK modulation, $\phi_{0}=45^{\circ}$, $\operatorname{SNR}\left(\phi_{0}\right)=21 \mathrm{~dB}, P E_{D D M}=50 \%$. 


\subsection{Zero Mean Artificial Non-Gaussian Noise: Results and Discussion}

In this subsection, we continue to use the synthesis method of [19], but we set the artificial noise to constant magnitude and random phase, as proposed in $[16,23,24]$, so that $P E_{D D M}$ can be adjusted easily based on (8). In that case, the injected artificial noise is non-Gaussian and the constellation symbol cluster is constrained inside a circle (Figure 5). Therefore, there may be some observation angles where the total noise $\left(r p_{a v_{-} W}(\phi)+N_{A W G N}\right)$ does not follow a Gaussian distribution. Figure 6 shows the results of BER for a DDM configuration where the use of (12) and expressions in Table 2 fails to predict accurately BER in the side lobes and the edges of the information beam. Those observation angles satisfy

$$
r p_{a v \_c o n v}(\phi) \gg r p_{a v \_W}(\phi)
$$

and

$$
r p_{a v_{-} W}(\phi)>N_{A W G N} \text {. }
$$

Nevertheless, it must be noted that these conditions are very particular (low $r p_{a v}{ }_{W}(\phi)$ and low $N_{A W G N}$ ) and do not apply in most practical scenarios. Indeed, if we test again all the configurations of Figure 2 using zero mean artificial non-Gaussian noise, we obtain the same results of Figure 3 because (13) and (14) are not satisfied at any observation angle. However, we are still spreading the artificial noise uniformly in all the observation angles out of the main beam, so the synthesis method is still inefficient in terms of $P E_{D D M}$.

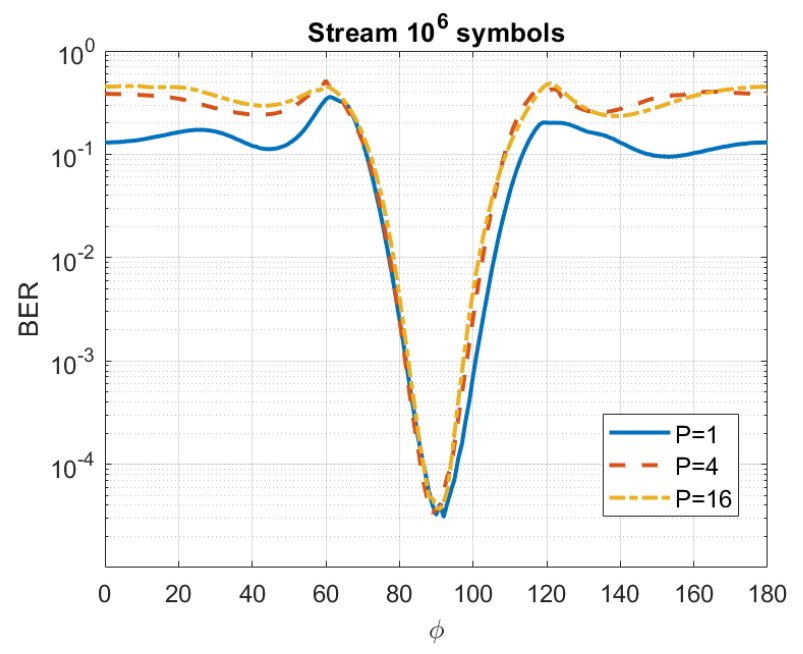

(a)

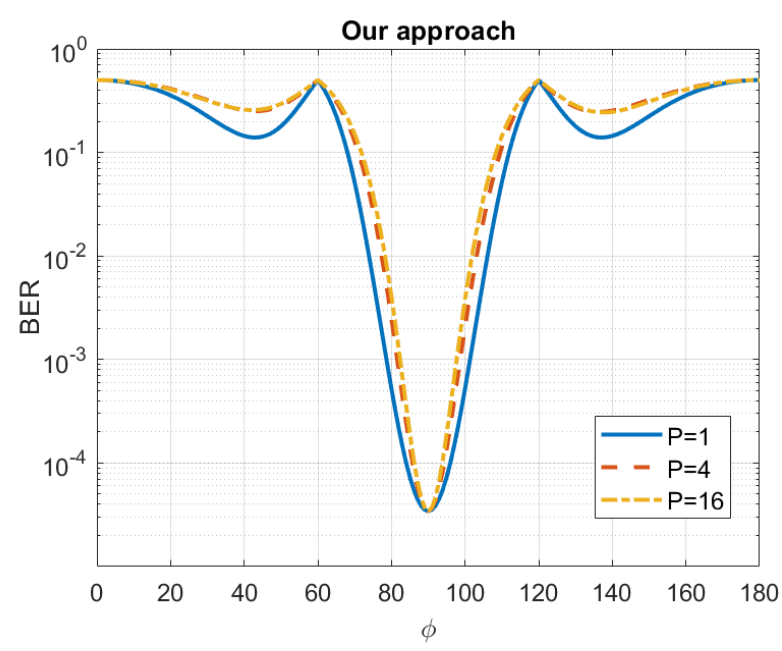

(b)

Figure 4. Array of four omnidirectional antennas along $x$-axis, spacing $\lambda / 2$. BER in $X Y$ plane for DDM and different number of available radiation patterns per symbol $(P)$. QPSK modulation, $\phi_{0}=90^{\circ}, \operatorname{SNR}\left(\phi_{0}\right)=12 \mathrm{~dB}, P E_{D D M}=75 \%$. Zero mean artificial Gaussian noise. Only half-plane is plotted due to symmetry. (a) Stream of $10^{6}$ symbols. (b) Our approach. 


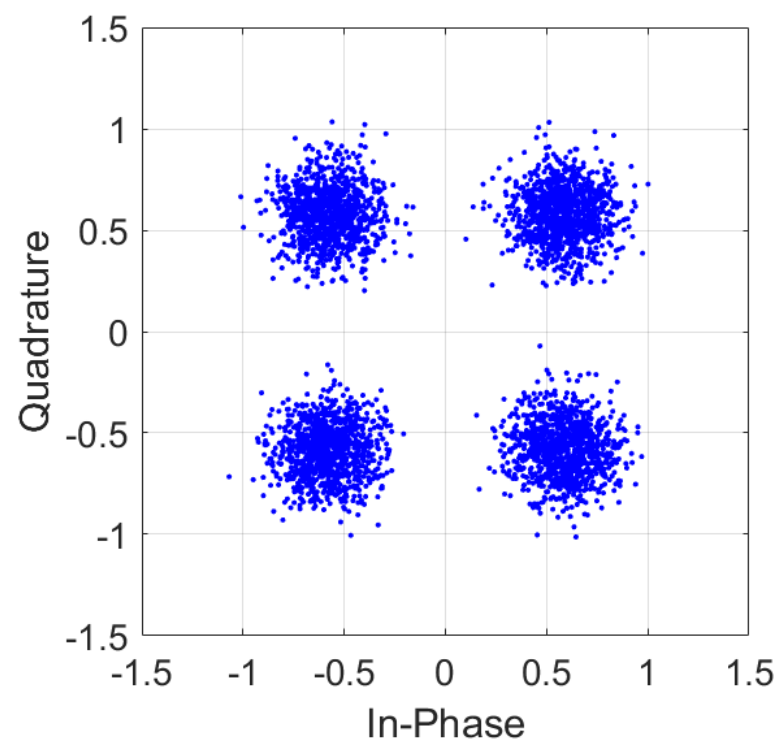

(a)

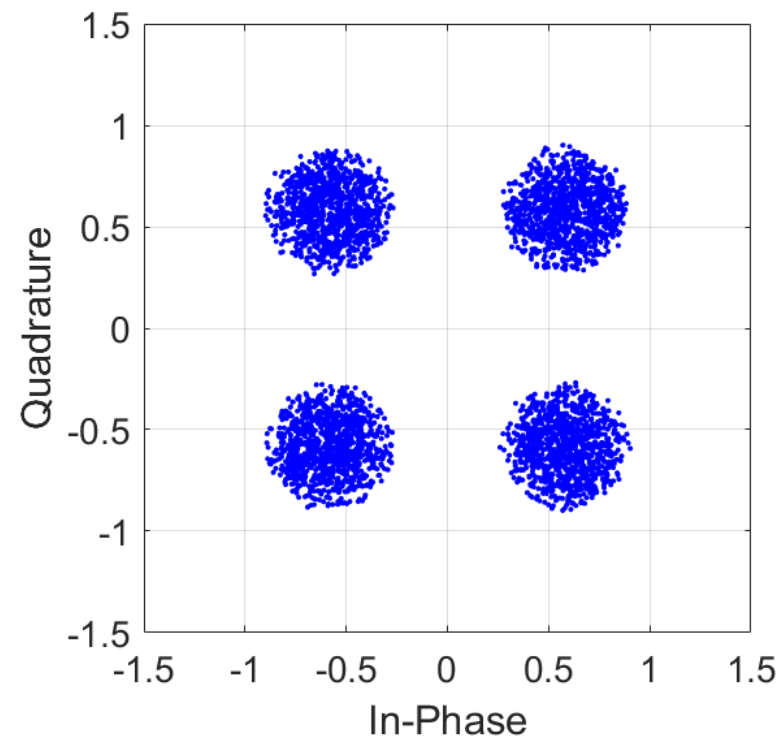

(b)

Figure 5. Array of four omnidirectional antennas along the $x$-axis, spacing $\lambda / 2$. QPSK modulation, $\phi_{0}=90^{\circ}, \operatorname{SNR}\left(\phi_{0}\right)=\infty$, $P E_{D D M}=75 \%, P=1024$. Transmitted symbols for DDM in observation angle $\phi=80^{\circ}$. (a) Zero mean artificial Gaussian noise. (b) Zero mean artificial Non-Gaussian noise (constant magnitude and random phase).

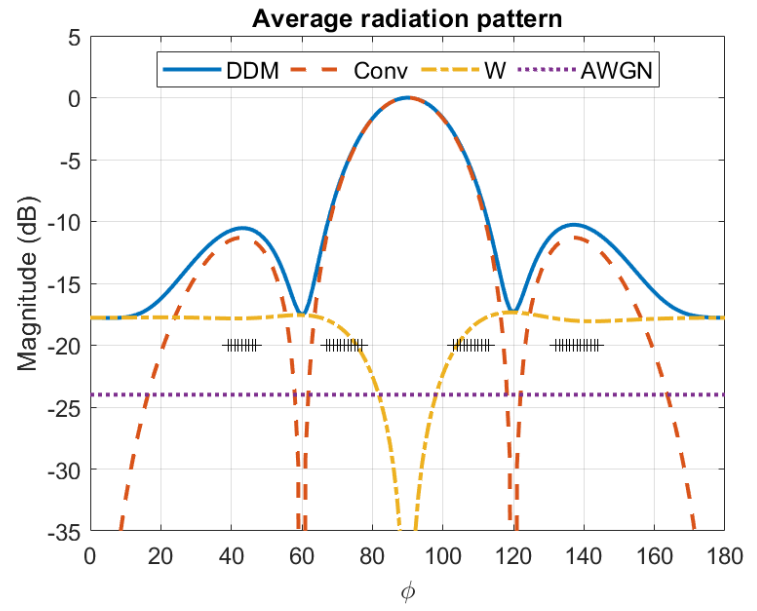

(a)

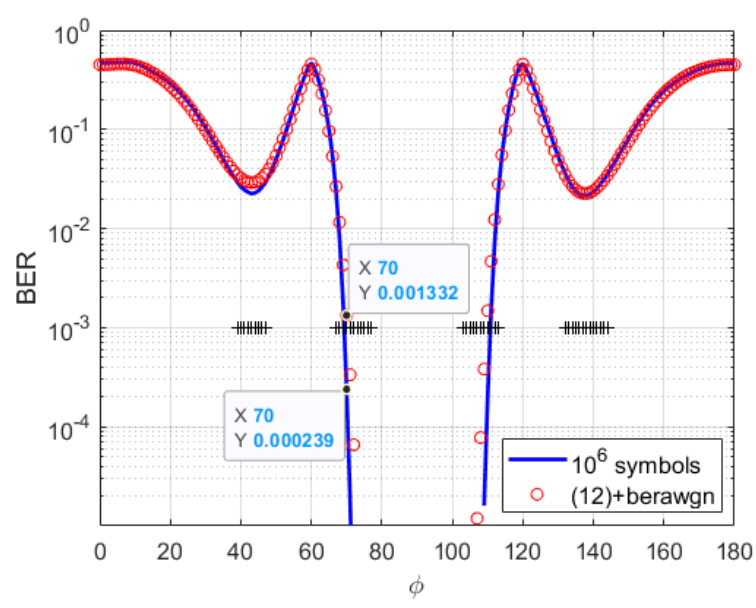

(b)

Figure 6. Array of four omnidirectional antennas along $x$-axis, spacing $\lambda / 2$. Average radiation patterns (a) and BER (b) in $X Y$ plane for DDM. Zero mean artificial Non-Gaussian noise. QPSK modulation, $\phi_{0}=90^{\circ}, \mathrm{SNR}\left(\phi_{0}\right)=24 \mathrm{~dB}, P E_{D D M}=95 \%$, $P=64$. Only half plane is plotted due to symmetry. Black crosses mark those observation angles where (13) and (14) are satisfied and our approach may fail to predict accurately BER.

\section{Non-Iterative BER-Driven DDM Synthesis}

In this section, we use the concepts previously developed to perform a non-iterative BER-driven DDM synthesis.

\subsection{General Procedure}

We start by selecting the modulation scheme, the antenna array $(\operatorname{AEP}(\phi))$, the secure observation angle $\phi_{0}$ and $\operatorname{BER}\left(\phi_{0}\right)$. We apply (2) to derive $\mathbf{B}_{m_{-}}$conv and, thereafter, (1) and (10) to evaluate $r p_{a v_{-} c o n v}(\phi)$. Taking into consideration that, at $\phi_{0}$, zero-mean 
AWGN is the only noise contribution, we derive $S N R_{a v}\left(\phi_{0}\right)$ using $\operatorname{BER}\left(\phi_{0}\right)$, the modulation scheme, and [36], subsequently, we apply (12) to obtain $N_{A W G N}$.

Secondly, for $K$ observation angles $\phi_{k}$, we define restrictions $\operatorname{BER}\left(\phi_{k}\right)$. Assuming that the total noise distribution at $\phi_{k}$ follows a zero mean Gaussian distribution, $S N R_{a v}\left(\phi_{k}\right)$ is calculated using $\operatorname{BER}\left(\phi_{k}\right)$, the modulation scheme and [36]. Then, we use (12) to obtain $r p_{a v_{-} W}\left(\phi_{k}\right)$.

Next, we compute column vectors $\mathbf{C}_{k}$ with the antenna weights that generate an interference radiation pattern with the main beam pointing to $\phi_{k}$

$$
\mathbf{C}_{k}=\operatorname{AEP}\left(\phi_{k}\right)^{*} /\left\|\operatorname{AEP}\left(\phi_{k}\right)\right\| \quad k \in\{1 \ldots K\} .
$$

In general, the interference radiation patters generated with $\mathbf{C}_{k}$ will be also present in the secure observation angle $\phi_{0}$. Therefore, these antenna weights need to be modified to steer a null of the interference radiation pattern along $\phi_{0}$. According to the power pattern projection method in [23], the antenna weights $\mathbf{A}_{k}$ that produce the desired radiation patterns can be obtained from $\mathbf{C}_{k}$ as

$$
\mathbf{A}_{k}=\left(\mathbf{I}_{\mathbf{N}}-\left(\mathbf{B}_{m \_ \text {conv }}\right)^{-1} \mathbf{B}_{m \_ \text {conv }}\right) \mathbf{C}_{k} \quad k \in\{1 \ldots K\},
$$

where $\mathbf{I}_{\mathbf{N}}$ is the N-by-N identity matrix and ${ }^{-1}$ is the Moore-Penrose pseudoinverse.

Finally, the weights that produce DDM are obtained as

$$
\mathbf{B}_{m p \_D D M}=\mathbf{B}_{m_{-} \text {conv }}+\sum_{k=1}^{K} G_{k} e^{j \psi_{p k}} \mathbf{A}\left(\phi_{k}\right) \quad m \in\{1 \ldots M\}, \quad p \in\{1 \ldots P\},
$$

where $\psi_{p k}$ is a random phase with uniform distribution updated for every $p$ and $k$. $G_{k}$ is a constant to be computed in order to satisfy

$$
r p_{a v_{-} W}\left(\phi_{k}\right)=\left\|\operatorname{AEP}\left(\phi_{k}\right)^{T} \cdot G_{k} \mathbf{A}_{k}\right\|^{2} \quad k \in\{1 \ldots K\} .
$$

It must be noted that, after (17), the injected artificial orthogonal noise follows a zero-mean non-Gaussian distribution as in Figure $5 b$.

\subsection{Results: Synthesis Example}

Let us consider the compact multimode antenna proposed in [37]. This radiating structure can perform the functions of a four-element array while generating a radiation pattern with a single maximum that can be steered freely in any direction in the XY plane. A drawback of this antenna is the high side lobes of the radiation pattern, which may compromise security in those observation angles (Figure 7). This performance can be improved with DDM.

A QPSK modulation will be transmitted along the secure observation angle $\phi_{0}=180^{\circ}$ with $\operatorname{BER}\left(180^{\circ}\right)=3.43 \times 10^{-5}$. Figure 8 depicts the typical curve of BER as a function of $S N R$ for a QPSK modulation under the assumption of AWGN and conventional Gray coding [36], so we determine $S N R_{a v}\left(180^{\circ}\right)=12 \mathrm{~dB}$ and, considering (3), $N_{A W G N}=-12 \mathrm{~dB}$.

In order to improve the security of the transmission, let us suppose that we are interested in lowering $\mathrm{BER}$ in side lobes $\left(\mathrm{BER}\left(111^{\circ}\right) \approx 10^{-1}\right)$ and narrowing the information beam width $\left(\operatorname{BER}\left(190^{\circ}\right) \approx 10^{-3}\right)$. From Figure 8, we obtain $S N R_{a v}\left(111^{\circ}\right)=2 \mathrm{~dB}$ and $S N R_{a v}\left(190^{\circ}\right)=9.8 \mathrm{~dB}$, and, using (12) the power of the artificial noise to be injected gives $r p_{a v_{-} W}\left(111^{\circ}\right)=-4.5 \mathrm{~dB}$ and $r p_{a v_{-} W}\left(190^{\circ}\right)=-16.3 \mathrm{~dB}$. In this example, conditions (13) and (14) are satisfied in no observation angle; therefore, the total noise will approximately behave as a zero mean Gaussian distribution even if the artificial noise is non-Gaussian.

Figure 9 shows the interference radiation patterns generated with the antenna weights $C_{1}$ and $C_{2}$ also interfering the secure observation angle $\left(180^{\circ}\right)$. Therefore, these antenna weights need to be modified to steer a null of the interference radiation pattern along $180^{\circ}$. The interference radiation patterns obtained using the antenna weights $\mathbf{A}_{1}$ and $\mathbf{A}_{2}$ are 
also plotted in Figure 9. It must be noted that, after (16), we find a null at $180^{\circ}$, but the interference pattern maximum is not necessarily at $111^{\circ}\left(190^{\circ}\right)$. Next, we apply (18) to

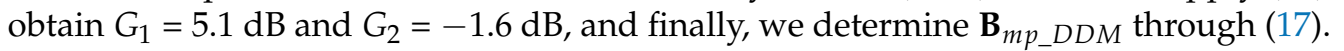

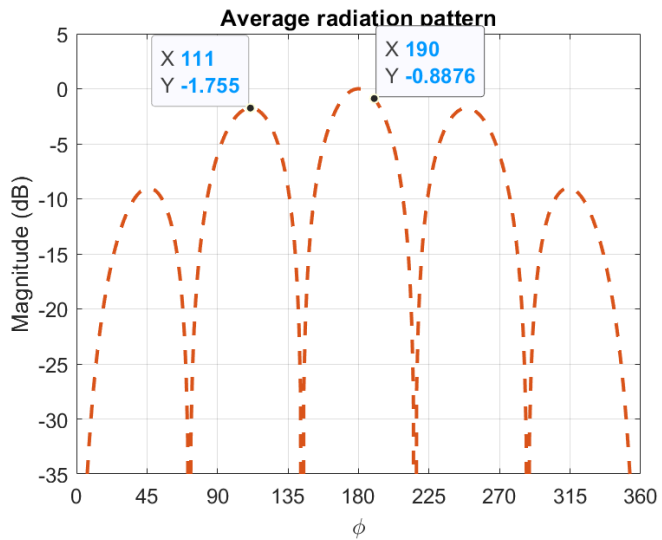

(a)

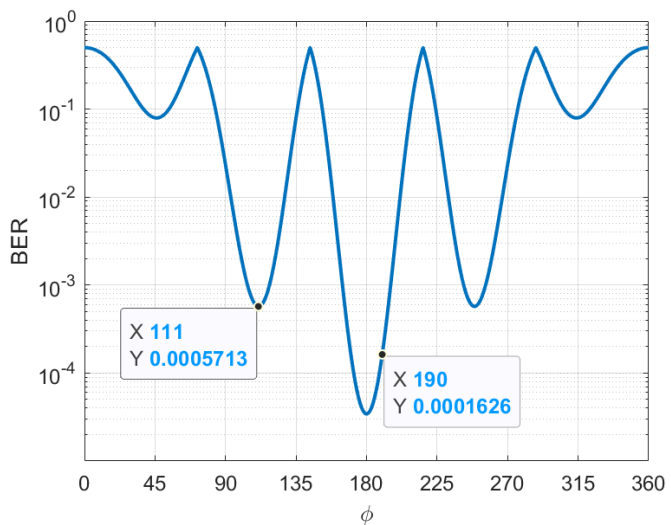

(b)

Figure 7. Compact antenna of [37]. QPSK modulation, $\phi_{0}=180^{\circ}, \mathrm{SNR}\left(\phi_{0}\right)=12 \mathrm{~dB}, P E_{D D M}=100 \%$ (conventional). Average radiation pattern $(\mathbf{a})$ and $\mathrm{BER}(\mathbf{b})$ in the $\mathrm{XY}$ plane.

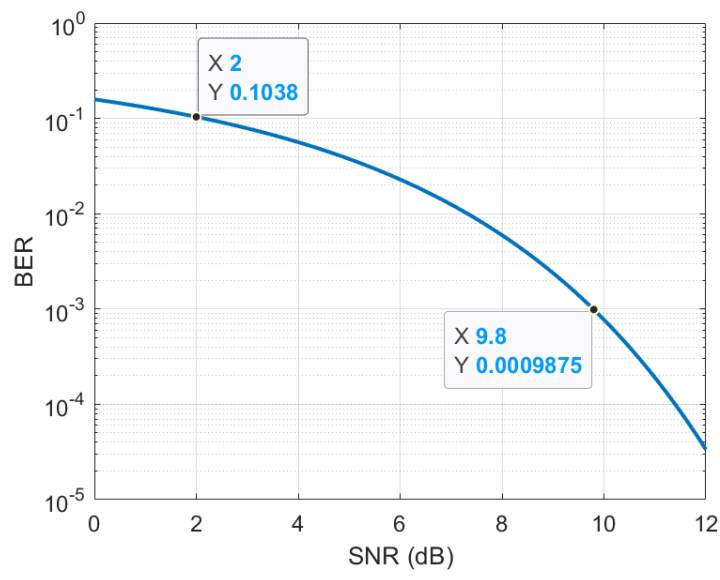

Figure 8. BER for QPSK modulation and Gray coding as a function of SNR.

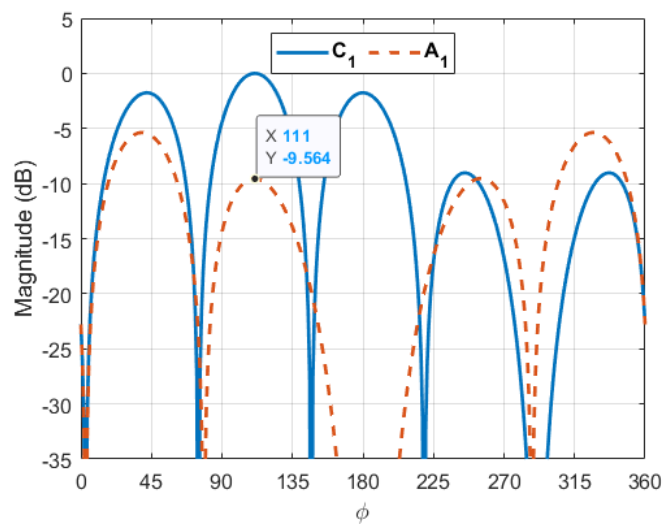

(a)

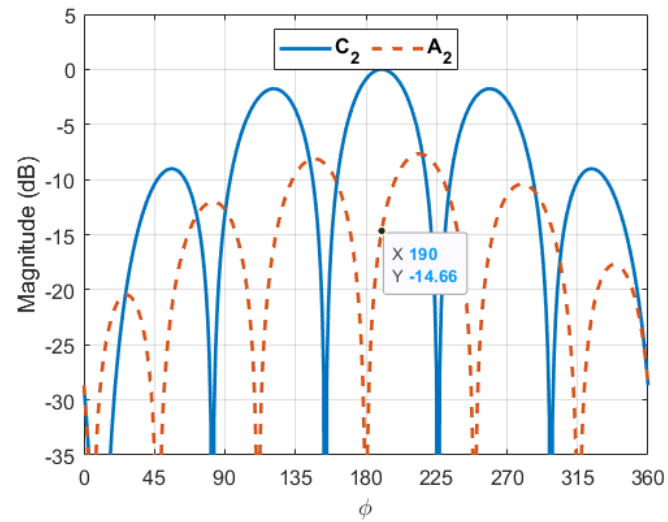

(b)

Figure 9. Compact antenna of [37], interference radiation patterns generated by antenna weights $\mathbf{C}_{k}(15)$ and $\mathbf{A}_{k}$ (16). (a) $\phi_{1}=111^{\circ}$. (b) $\phi_{2}=190^{\circ}$. 
The average radiation patterns obtained after our synthesis and the BER computed with our approach compared to the BER evaluated through the transmission of $10^{6}$ symbols can be found in Figure 10.

\subsection{Discussion}

As expected, the evaluation of BER using (12) overlaps with the results obtained from transmitting $10^{6}$ symbols, although the injected artificial orthogonal noise follows a zero-mean non-Gaussian distribution, as shown in Figure 10b .

In Figure 10a, we can observe that the injected artificial noise was adjusted precisely in the direction of side lobes, while reduced interference was introduced in the nulls of the conventional pattern (where no information was transmitted). The proposed specifications were achieved for $P E_{D D M}=45 \%$ without the need to follow an iterative procedure where BER needs to be evaluated many times. Surprisingly, the BER curve is almost symmetric, although the restrictions were not applied symmetrically. Another interesting feature of this synthesis procedure is that we have been able to adjust the BER performance for side lobes and the information beam width independently.

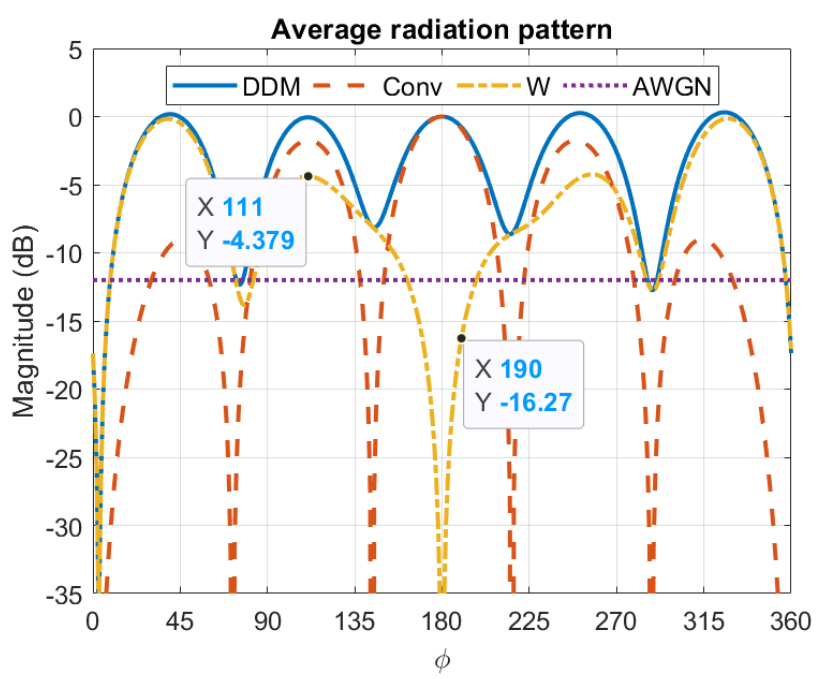

(a)

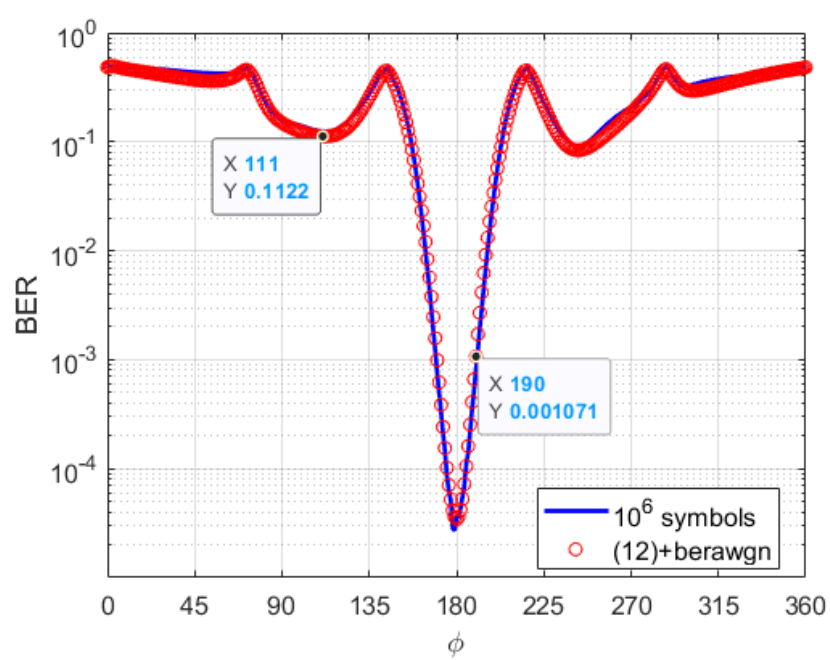

(b)

Figure 10. Compact antenna of [37]. QPSK modulation, $\phi_{0}=180^{\circ}, \mathrm{SNR}\left(\phi_{0}\right)=12 \mathrm{~dB}, P E_{D D M}=45^{\circ}, P=64$. Zero-mean artificial non-Gaussian noise. Average radiation pattern (a) and BER (b) in the XY plane.

\section{Conclusions}

Bit error rate is the most accepted figure of merit for evaluating the performance of DDM in terms of security. The transmission of a stream of symbols with AWGN for every observation angle is the most common procedure to evaluate BER in DDM, but it may be time-consuming. In this work, we have proven that, taking the orthogonal vector approach as a starting point and defining an average SNR appropriately, BER for standard constellations can be calculated accurately and efficiently using well-known theoretical expressions and/or computer codes.

Several experiments have been carried out to verify that the proposed solution perfectly predicts BER for different modulation schemes, efficiencies and SNR as long as the injected artificial noise follows a zero-mean Gaussian distribution. We have also shown that, under some general conditions that are not difficult to fulfill, our solution is still valid when the artificial noise distribution is zero-mean non-Gaussian.

The possibility of evaluating BER using only information from the transmitted radiation patterns also allows for a non-iterative synthesis of DDM imposing independent BER restrictions for the information beam width and the side lobes region. In this way, a more efficient use of the interfering power is achieved, since it is concentrated in the observation 
angles where the information is most vulnerable to potential eavesdroppers. A general DDM synthesis procedure has been presented and applied to improve the performance, in terms of security, of a multiport compact antenna with $360^{\circ}$ beam steering that generates a radiation pattern with very high side lobes.

Author Contributions: Conceptualization, J.P. and A.T.; methodology, J.P.; software, J.P. and E.C.-H.; validation, E.C.-H.; formal analysis, J.P.; investigation, J.P. and E.C.-H.; resources, J.P. and A.T.; writing — original draft preparation, J.P.; writing — review and editing, J.P., E.C.-H. and A.T.; visualization, J.P. and E.C.-H.; supervision, J.P. and A.T.; project administration, J.P.; funding acquisition, J.P. and A.T. All authors have read and agreed to the published version of the manuscript.

Funding: This work was supported by the Universitat Autonoma de Barcelona, FEDER funds and the Spanish Ministry of Science, Innovation and Universities (RTI2018-096019-B-C33).

Data Availability Statement: Data are contained within the article. Matlab codes used to generate the results are available from the corresponding author upon request.

Conflicts of Interest: The authors declare no conflict of interest.

\section{References}

1. Zou, Y.L.; Zhu, J.;Wang, X.B.; Hanzo, L. A survey on wireless security: technical challenges, recent advances, and future trends. Proc. IEEE 2016, 104, 1727-1765. [CrossRef]

2. Stallings, W. Cryptography and Network Security: Priciples and Practice, 7th ed.; Pearson Education Limited: Harlow, UK, 2017.

3. Barenghi, A.; Breveglieri, L.; Koren, I.; Naccache, D. Fault injection attacks on cryptographic devices: Theory, practice, and countermeasures. Proc. IEEE 2012, 100, 3056-3076. [CrossRef]

4. Mukherjee, A.; Fakoorian, SAAHuang, J.; Swindlehurst, A.L. Principles of physical layer security in multiuser wireless networks: A survey. IEEE Commun. Surv. Tutor. 2014, 16, 1550-1573. [CrossRef]

5. Chen, X.M.; Ng, D.W.K.; Gerstacker, W.H.; Chen, H.S. A survey on multiple-antenna techniques for physical layer security. IEEE Commun. Surv. Tutor. 2016, 19, 1027-1053. [CrossRef]

6. Sun, L.; Du, Q. A Review of Physical Layer Security Techniques for Internet of Things: Challenges and Solutions. Entropy 2018, 20, 730. [CrossRef]

7. Goel, S.; Negi, R. Guaranteeing secrecy using artificial noise. IEEE Trans. Wirel. Commun. 2008, 7, 2180-2189. [CrossRef]

8. Ding, Y.; Fusco, V. Directional-modulation-enabled physical-layer wireless security. In Trusted Communications with Physical Layer Security for 5 G and Beyond; Duong, T.Q., Zhou, X., Poor, H.V., Eds.; The Institution of Engineering and Technology: London, UK, 2017; pp. 313-336.

9. Ding, Y.; Fusco, V. Establishing metrics for assessing the performance of directional modulation systems. IEEE Trans. Antennas Propag. 2014, 62, 2745-2755. [CrossRef]

10. Babakhani, A.; Rutledge, D.B.; Hajimiri, A. Near-field direct antenna modulation. IEEE Microw. Mag. 2009, 10, 36-46. [CrossRef]

11. Daly, M.; Bernhard, J. Directional modulation technique for phased arrays. IEEE Trans. Antennas Propag. 2009, 57, 2633-2640. [CrossRef]

12. Daly, M.P.; Daly, E.L.; Bernhard, J.T. Demonstration of directional modulation using a phased array. IEEE Trans. Antennas Propag. 2010, 58, 1545-1550. [CrossRef]

13. Daly, M.P.; Bernhard, J.T. Beamsteering in pattern reconfigurable arrays using directional modulation. IEEE Trans. Antennas Propag. 2010, 58, 2259-2265. [CrossRef]

14. Hong, T.; Song, M.Z.; Liu, Y. Dual-beam directional modulation technique for physical-layer secure communication. IEEE Antennas Wirel. Propag. Lett. 2011, 10, 1417-1420. [CrossRef]

15. Zhang, Y.; Ding, Y.; Fusco, V.Sidelobe modulation scrambling transmitter using Fourier Rotman lens. IEEE Trans. Antennas Propag. 2013, 61, 3900-3904. [CrossRef]

16. Ding, Y.; Zhang, Y.; Fusco, V. Fourier Rotman lens enabled directional modulation transmitter. Int. J. Antennas Propag. 2015, 2015, 1-13. [CrossRef]

17. Valliappan, N.; Lozano, A.; Heath, R.W. Antenna subset modulation for secure millimeter-wave wireless communication. IEEE Trans. Commun. 2013, 61, 3231-3245. [CrossRef]

18. Ding, Y.; Fusco, V. BER-driven synthesis for directional modulation secured wireless communication. Int. J. Microw. Wirel. Technol. 2013, 6, 139-149. [CrossRef]

19. Ding, Y.; Fusco, V.F. A vector approach for the analysis and synthesis of directional modulation transmitters. IEEE Trans. Antennas Propag. 2014, 62, 361-370. [CrossRef]

20. Shi, H.; Tennant, A. Simultaneous, multichannel, spatially directive data transmission using direct antenna modulation. IEEE Trans. Antennas Propag. 2014, 62, 403-410. [CrossRef]

21. Zhu, Q.; Yang, S.; Yao, R.; Nie, Z. Directional modulation based on 4-D antenna arrays. IEEE Trans. Antennas Propag. 2014, 62, 621-628. [CrossRef] 
22. Ding, Y.; Fusco, V.F. Constraining directional modulation transmitter radiation patterns. IET Microw. Antennas Propag. 2014, 8, 1408-1415. [CrossRef]

23. Ding, Y.; Fusco, V.F. Directional modulation far-field pattern separation synthesis approach. IET Microw. Antennas Propag. 2015, 9 , 41-48. [CrossRef]

24. Ding, Y.; Fusco, V.F. MIMO-inspired synthesis of directional modulation systems. IEEE Antennas Wirel. Propag. Lett. 2016, 15, 580-584. [CrossRef]

25. Hu, J.; Shu, F.; Li, J. Robust synthesis method for secure directional modulation with imperfect direction angle. IEEE Commun. Lett. 2016, 20, 1084-1087. [CrossRef]

26. Ding, Y.; Fusco, V. A review of directional modulation technology. Int. J. Microw. Wirel. Technol. 2015, 8, 981-993. [CrossRef]

27. Narbudowicz, A.; Amman, M.J.; Heberling, D. Directional modulation for compact devices. IEEE Antennas Wirel. Propag. Lett. 2017, 16, 2094-2097. [CrossRef]

28. Ding, Y.; Fusco, V. A synthesis-free directional modulation transmitter using retrodirective array. IEEE J. Sel. Top. Signal Process. 2017, 11, 428-441. [CrossRef]

29. Sun, C.; Yang, S.; Chen, Y.; Guo, J.; Qu, S.; Hu, J. 4-D retro-directive antenna arrays for secure communication based on improved directional modulation. IEEE Trans. Antennas Propag. 2018, 66, 5926-5933. [CrossRef]

30. Hong, T.; Shi, X.P.; Liang, X.S. Synthesis of sparse linear array for directional modulation via convex optimization. IEEE Trans. Antennas Propag. 2018, 66, 3959-3972. [CrossRef]

31. Zhang, B.; Liu, W.; Li, Y.; Zhao, X.; Wang, C. Directional modulation design under a constant magnitude constraint for weight coefficients. IEEE Access 2019, 7, 154711-154718. [CrossRef]

32. Zhang, B.; Liu, W.; Li, Y.; Zhao, X.; Wang, C. Directional modulation design under maximum and minimum magnitude constraints for weight coefficients. Ad Hoc Netw. 2020, 10, 102034. [CrossRef]

33. Pozar, D. The active element pattern. IEEE Trans. Antennas Propag. 1994, 42, 1176-1178. [CrossRef]

34. Proakis, J.; Salehi, M. Digital Communications, 5th ed.; McGraw-Hill Education: New York, NY, USA, 2008 ; pp. 160-289.

35. Cho, K.; Yoon, D. On the general BER expression of one- and two-dimensional amplitude modulations. IEEE Trans. Commun. 2002, 50, 1074-1080.

36. Bit Error Rate, Matlab Help Centre. Available online: https://es.mathworks.com/help/comm/ug/bit-error-rate-ber.html\#bq421 b1 (accessed on 13 January 2021).

37. Parron, J.; Cabrera-Hernandez, E.A.; Tennant, A.; DePaco, P. Multiport compact stacked patch antenna with $360^{\circ}$ beam steering for generating dynamic directional modulation. IEEE Trans. Antennas Propag. 2021, 69, 1162-1167. [CrossRef] 